\title{
Amorphous Silicon Based Particle Detectors
}

\author{
N. Wyrsch ${ }^{1}$, A. Franco ${ }^{1}$, Y. Riesen ${ }^{1}$, M. Despeisse ${ }^{1}$, S. Dunand ${ }^{1}$, F. Powolny ${ }^{2}$, P. Jarron ${ }^{2}$ and C. \\ Ballif $^{1}$ \\ ${ }^{1}$ Ecole Polytechnique Fédérale de Lausanne (EPFL), Institute of Microengineering (IMT), \\ ${ }^{2}$ Photovoltaics and thin film electronics laboratory, Breguet 2, 2000 Neuchâtel, Switzerland. \\ CERN, CERN Meyrin, 1211 Genève 23, Switzerland.
}

\begin{abstract}
Radiation hard monolithic particle sensors can be fabricated by a vertical integration of amorphous silicon particle sensors on top of CMOS readout chip. Two types of such particle sensors are presented here using either thick diodes or microchannel plates. The first type based on amorphous silicon diodes exhibits high spatial resolution due to the short lateral carrier collection. Combination of an amorphous silicon thick diode with microstrip detector geometries permits to achieve micrometer spatial resolution beneficial for high accuracy beam positioning. Microchannel plates based on amorphous silicon were successfully fabricated and multiplication of electrons was observed. This material may solve some of the problems related to conventional microchannel devices. Issues, potential and limits of these detectors are presented and discussed.
\end{abstract}

\section{INTRODUCTION}

Hydrogenated amorphous silicon ( $\mathrm{a}-\mathrm{Si}: \mathrm{H})$ exhibits two advantages for applications as particle sensors: it is one of the most radiation hard semiconductors [1] and can be deposited as thin layers on various types of substrates over large areas. For these reasons this material has attracted much attention for direct and indirect (using a scintillating layer) particle detection [2]. Using a direct detection scheme, various types of particles such as protons, neutron, electrons or $\mathrm{X}$-ray were successfully detected using thick a-Si:H diode $[3,4,5]$. Due to the low interaction of minimum ionizing particle (MIP) with silicon (due to the low atomic mass of this material) very thick diodes are necessary. Given the high voltage needed to fully deplete the device, such thick diodes are challenging in terms of the fabrication process and of the quality the material.

While a-Si:H has been successfully implemented in indirect particle detectors for X-ray radiography [6], a Si:H detector has never been practically used for direct particle detection in a physics experiment or in a commercial product. Insufficient performance, failure to detect single MIP, metastability of a-Si:H and fabrication issues are some of the reasons for this lack of success. Nevertheless, as demonstrated here, a-Si:H thick diodes can be improved for MIP detection and selected applications could greatly benefit from present a-Si:H based detectors. Vertical integration of a thick a Si:H diode array on top of a readout ASIC (so-called "thin-film on ASIC" or "TFA" technology) (see Fig. 1) enables the fabrication of very radiation hard detector and may help achieving single MIP detection [7]. Using microstrip pixel geometries, very high spatial resolution can be obtained that are comparable or superior to the ones of stateof-the-art c-Si detectors. 

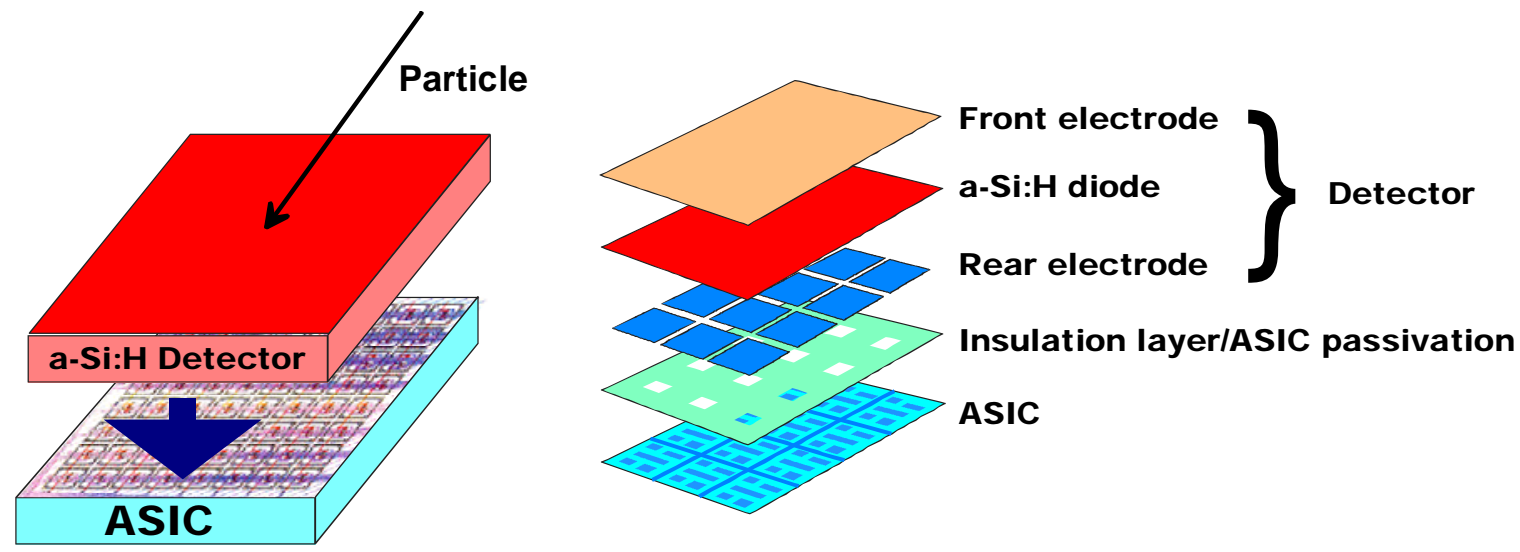

Figure 1. Schematic view of a thin-film on ASIC (TFA) particle detector.

A specific test chip was designed in the past by CERN to study the effect of pixel geometry on the performance of TFA sensors [8]. This chip includes pixels of various sizes and shapes, microstrips of various width and pitch values and two different geometries for the openings in the passivation layer of the chip (see Fig. 2). With this chip (as well as for most TFA devices), the back contacts of the individual pixels are provided by the last metal layer of the ASIC through openings in the ASIC passivation. The latter can be local or global. For the local case, the opening corresponds to the size of the metal pad with a positive or negative overlap (the size of the opening being slightly larger or slightly smaller than the size of the local metal pad). A global opening corresponds to a case where we have a single opening for several metal pads or pixels. For all microstrips present on this test chip, the openings are global. Several studies have demonstrated that the edges from the passivation openings lead to important leakage current at the periphery of the pixels in case of local openings. Global opening is therefore necessary to keep low leakage current values $[9,10]$. In the same studies, it was also demonstrated that diode without n-layer (i.e. an i-p layer structure directly deposited on the metallic back contact -i.e. metal-i-p configuration) further reduces the leakage. In this paper, the spatial response of microstrips and the carrier collection is analyzed for various strip and diode geometries and diode configurations.

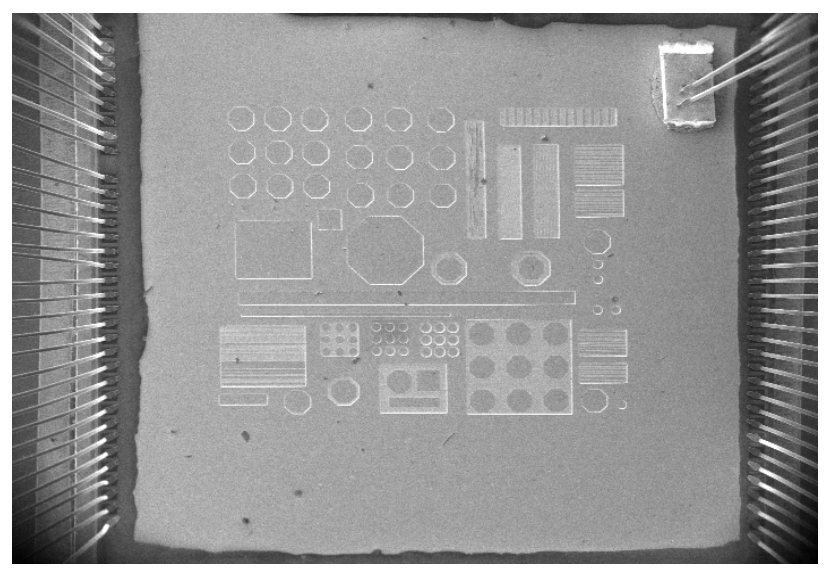

Figure 2. Picture of a $4 \times 4 \mathrm{~mm}^{2}$ test circuit (aSiHTest) designed for TFA performance analysis coated with a thick a-Si:H n-i-p diode. Various pixel and microstrip structures can be seen. 
We recently proposed microchannel plate (MCP) detectors based on a-Si:H as an alternative to particle detection using thick diodes $[11,12]$. Such detectors consist of thick plates with very narrow micro-channels drilled throughout the device. When a primary electron hits the channel wall secondary electrons are emitted. Since a high electric field is applied between the two faces of the plate, an avalanche mechanism takes place in the microchannels. These avalanches thus lead to multiplication of the primary electrons. MCP are commonly used as image intensifying devices [13]. For MCP, a-Si:H material properties permit to overcome some performance limitations imposed by the lead glass or c-Si material used in commercial MCP fabrication $[14,15]$.a-Si:H technology would also greatly simplify the fabrication of MCPs, would allow a vertical integration of such detectors on the readout electronics, thus broadening the range of applications. The typical structure of an AMCP (a-Si:H MCP) and its operation principle is given in Fig. 3. Issues regarding the fabrication of AMCP and latest characterization using EBIC techniques will be presented and discussed.

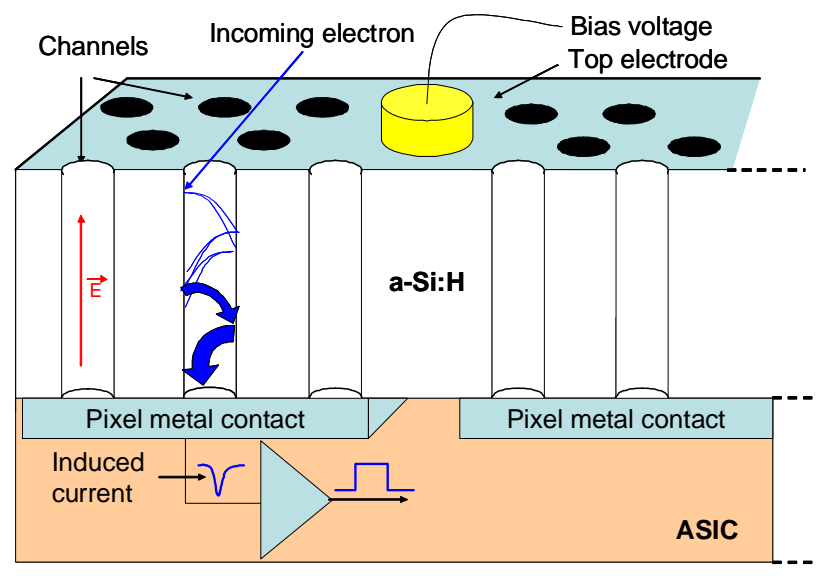

Figure 3. Schematic view of an a-Si:H based MCP (AMCP) structure, vertically integrated on a readout ASIC.

\section{EXPERIMENT}

Deposition of thick a-Si:H layers (as needed for the devices discussed here) requires careful optimization in order to achieve high deposition rate with reasonable material quality while maintaining low internal mechanical stress. In this context VHF PECVD (very high frequency plasma enhanced chemical vapor deposition) enables such deposition [16] and was used for the fabrication of all thick a-Si:H based devices discussed in this paper. Depositions were performed at a plasma excitation frequency of $70 \mathrm{MHz}$ and at deposition temperatures between 200 and $235^{\circ} \mathrm{C}$. While almost stress free layer can be deposited at temperatures around $170^{\circ} \mathrm{C}$ on c-Si [16], higher deposition temperature was needed to keep the film integrity and to avoid delamination during and after the growth for film thicknesses up to $100 \mu \mathrm{m}$ while obtaining acceptable compressive stress in the material. Typical deposition rate of $2 \mathrm{~nm} / \mathrm{s}$ was achieved during deposition.

All optimizations of thick diodes were performed on Cr-coated glass substrates with patterned ITO top contacts. Identical process parameters were used for the fabrication of all TFA devices. In this case the Al pads of the CMOS readout chips were used as the diode array back 
contacts and an ITO layer was used as common top contact. Diodes without n-layer were also used both in test and TFA devices. For metal-i-p (M-i-p) TFA configurations (using the chip metal pads as metal back contacts), a chemical cleaning of the CMOS pads was necessary to obtain reproducible and uniform device performance.

AMCP test devices were fabricated on oxidized 4" Si wafers with an oxide layer thickness of 1-2 $\mu \mathrm{m}$ covered with the Al back contact plane. $80-100 \mu \mathrm{m}$ thick a-Si:H intrinsic layer was then deposited followed by a 0.1-0.5 $\mu \mathrm{m}$ thick n-doped a-Si:H layer used as a common top contact of the MCP pixels. A mesa patterning of the a-Si:H layers was then performed by reactive ion etching (RIE) using a Cr hard mask before the deep reactive ion etching (DRIE) of the micro-channels. For some AMCP an additional $\mathrm{SiO}_{2}$ was deposited between the Al contact layer and the a-Si:H thick layer as an etch stop layer to improve the quality of the fabricated devices. Additional information on the fabrication of the AMCP as well as the measurement configuration can be found in [12].

An EBIC characterization system was set up in a commercial Philips ESEM XL30 (Environmental Scanning Electron Microscope) allowing electron beam energy values ranging from 1 to $30 \mathrm{keV}$. Simulations of the electron beam interaction with the a-Si:H based device were done using CASINO [17]. At $30 \mathrm{keV}$, the lateral spread of the electrons (coming from the beam) inside the a-Si:H layer was found to be $<1 \mu$ m while the maximum stopping power as a function of penetration of the electron beam is attained at the depth of $2 \mu \mathrm{m}$ and most of the electron energy is transferred to the material within $5 \mu \mathrm{m}$. The generation rate of electron-hole pairs (resulting from the energy loss from the electron beam) in this volume is estimated to be larger than $10^{22} \mathrm{~cm}^{-3} \mathrm{~s}^{-1}$.

\section{RESULTS AND DISCUSSION}

\section{$\underline{\text { TFA sensors - thick diode optimization }}$}

The intrinsic layer of the diode used for the collection of the electro-hole pairs generated by the ionizing particle should be fully depleted. The externally applied field should be high enough to allow the depletion region to extend through the entire intrinsic layer and to maximize charge collection. The depletion region is the active part of the diode. The voltage needed for full depletion increases as the square root of the thickness and voltage of $\approx 600 \mathrm{~V}$ is necessary to deplete a $30 \mu \mathrm{m}$ thick diode [18]. Practically one usually observes a supra linear increase of the diode leakage current as the voltage is raised and this effect is more pronounced when the diode thickness is increased. Concentration of the internal field at the p-i interface, Poole-Frenkel effect, and additional current injection from the p-layer are the main reasons for such an increase in the leakage current [7].

In a previous study [18] we showed that the introduction of a buffer layer at the p-i interface is, to some extend, effective at limiting the strong increase of the leakage current with bias voltage. However, leakage is in most cases still too high to get any benefit from diodes with thicknesses larger than $15 \mu \mathrm{m}$; the required voltage for full depletion leads to unacceptably high leakage current.

Recently p-doped $\mathrm{SiO}_{x}$ layers have been introduced in solar cells $[19,20]$. Such layers enable the quenching of local shunts by introducing an anistropic resistive layer between the contacts and the intrinsic layer. This material consists of c-Si filaments embedded into a silicon 
oxide matrix. It exhibits a much higher in plane resistivity than its transversal resistivity, therefore limiting the spatial spreading of the effect of shunts. Such layers have been introduced in thin $(1-2 \mu \mathrm{m})$ and thick $(10 \mu \mathrm{m})$ sensors. As one can observe in Fig. 4, such p-doped $\mathrm{SiO}_{\mathrm{x}}$ layers are effective at reducing current leakage in thin diodes. However, for thick ones, the benefit is not yet clear. Further study will be needed to clarify this observation.

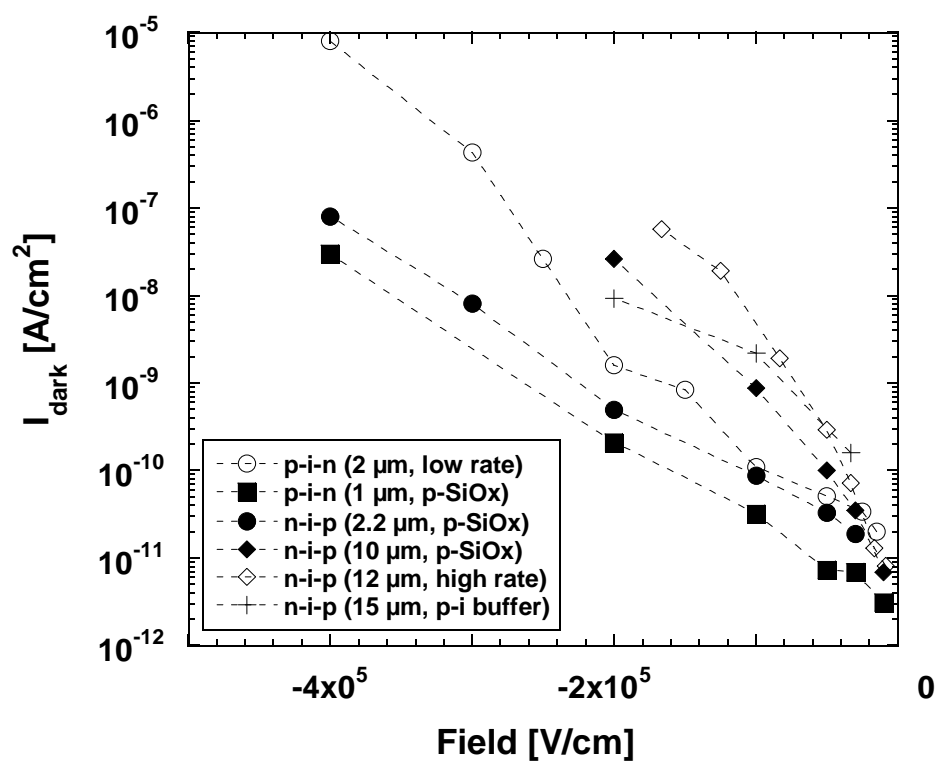

Figure 4. Dark current (or leakage current) of dioded of various thicknesses as a function of electrical field values. Full symbols correspond to diode incorporating a p-doped $\mathrm{SiO}_{\mathrm{x}}$ layer.

\section{TFA sensors - microstrip characterization}

Preliminary characterizations of the microstrips present in the chip shown in Fig. 2 were already performed using beta particles [8]. It was demonstrated that almost no cross-tall takes place for microstrips only $4 \mu \mathrm{m}$ apart and that the lateral collection of the charges generated by the ionizing particles is very short. Such microstrip structures could therefore permit very high spatial resolution, unmatched by the state-of-the-art detector technologies. In the case of c-Si based technology, localization of a beam/particle is given by measuring the charge sharing between two adjacent strips or pixels. The transport and collection is provided by diffusion process over relatively large distance of several tens or hundreds of microns. In the case of $\mathrm{a}-\mathrm{Si}: \mathrm{H}$, collection is ensured by drift process and therefore an electric field must be present. The electric field outside the pixels or microstrip is low and that explains the low lateral collection. The spatial resolution is therefore strongly linked to the density of the pixels or microstrips.

To get more insight about the lateral collection of carriers in a-Si:H based microstrips, various microstrips of the chip presented above were characterized by EBIC on several diode thicknesses and geometries. In Fig. 5, the SEM picture (a) and the corresponding EBIC image (b) are shown for microstrips with a width of $16.5 \mu \mathrm{m}$, spacing of $33.5 \mu \mathrm{m}$ and a-Si:H p-i-n diode of $5 \mu \mathrm{m}$. A one dimensional EBIC scan through the microstrips is also plotted on Fig. 6 . All microstrips of this structure are connected in parallel except the central one. One can observe that the charge collection between the strips is very limited, as expected. The dark lines in the EBIC pictures are due to previous line scan through the microstrips which locally degraded the material 
(due to very high generation rate of electron-hole pairs induced by the SEM electron beam) and affected charge collection. This degradation, similar to the effect of light-soaking in a-Si:H, is reversible and the initial state of the diode can be recovered by thermal annealing. The fluctuation of the signal "on the microstrips" (peaks of the signal in Fig. 6) are caused by local non uniformities that can also be observed in detailed EBIC maps or SEM pictures.
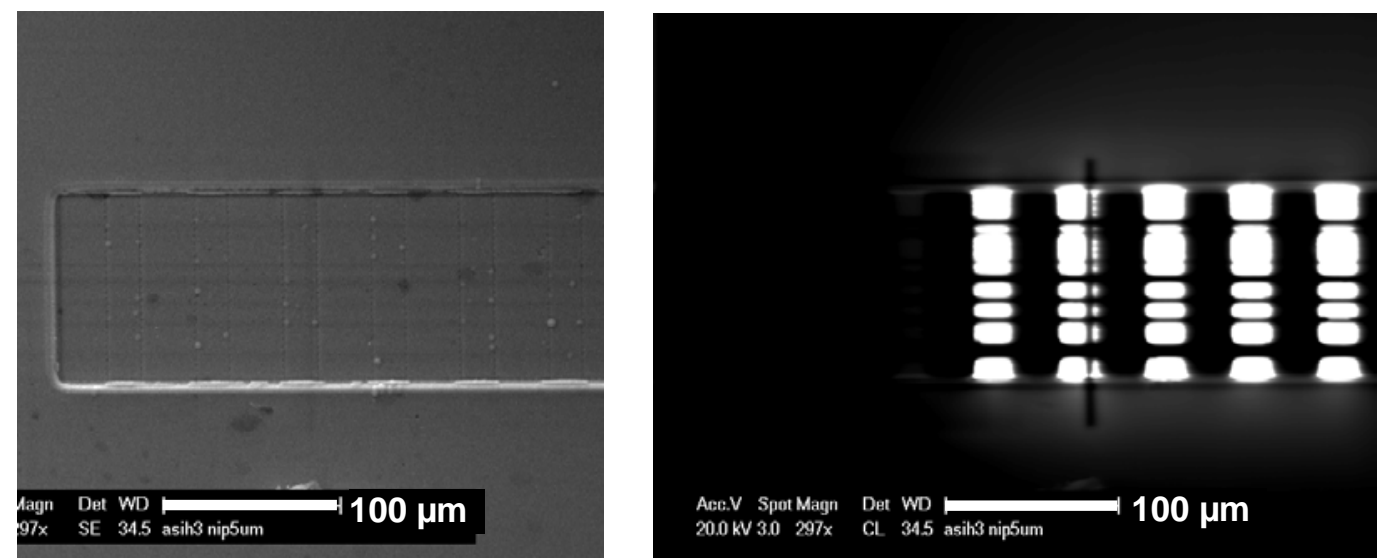

Figure 5. (Left) SEM image of a set of microstrips with a width of $16.5 \mu \mathrm{m}$ and spacing of 33.5 $\mu \mathrm{m}$ covered with $5 \mu \mathrm{m}$ a-Si:H n-i-p diode; (right) corresponding EBIC image obtained at applied voltage of $30 \mathrm{~V}$ and beam energy of $20 \mathrm{keV}$. All strips are connected in parallel except the left one. Dark regions in the EBIC image of the strips correspond to degraded area by previous EBIC line scans.

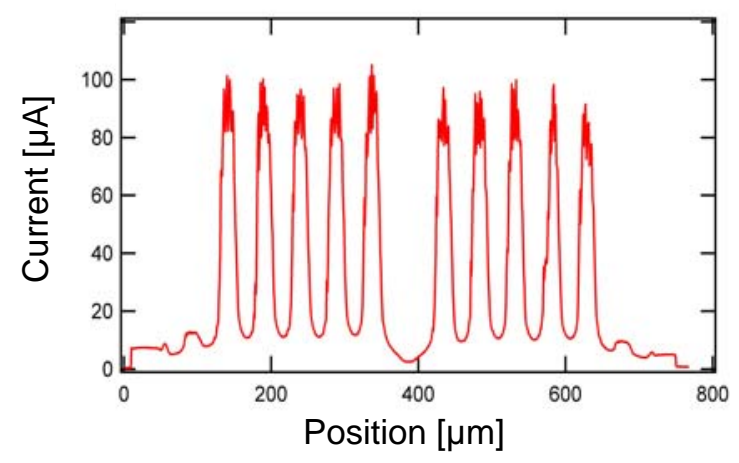

Figure 6. Signal from the EBIC line scans through the microstrip set of Fig. 5.

For detailed analysis, a Lateral Charge Collection ( $L C C)$ value was defined as

$$
L C C[\%]=\frac{\left(I_{\text {res }}-I_{\text {noise }}\right)}{\left(I_{\text {strip }}-I_{\text {noise }}\right)} \cdot 100
$$

where $I_{\text {strip }}$ is the average signal on a strip, $I_{\text {res }}$ the signal measured at mid-point between two microstrips, and $I_{\text {noise }}$ the residual signal far from a strip. A signal/noise ratio $(S / N)$ value was also defined as

$$
\frac{S}{N}=\frac{\left(I_{\text {strip }}-I_{\text {noise }}\right)}{I_{\text {noise }}}
$$


For the same diode thickness and configuration, EBIC pictures and one dimensional scan for microstrip with a width of $6.6 \mu \mathrm{m}$ and spacing of $16.4 \mu \mathrm{m}$ and for microstrips with a with of $1.5 \mu \mathrm{m}$ width and spacing of $3.5 \mu \mathrm{m}$ are shown in Fig. 7 and Fig. 8, respectively. $L C C$ and $S / N$ values measured on various microstrips are given in Table 1 . One can see that with narrower strips and smaller spacing the contrast between $I_{\text {strip }}$ and $I_{\text {res }}$ is reduced due to the fact that some charge is collected by the adjacent microstrips for smaller spacing. Nevertheless, the individual microstrips with $1.5 \mu \mathrm{m}$ width and only $3.5 \mu \mathrm{m}$ spacing can be easily resolved, both in the maps and one dimensional scans. This demonstrates that almost micrometric spatial resolution can be achieved with a-Si:H microstrips.
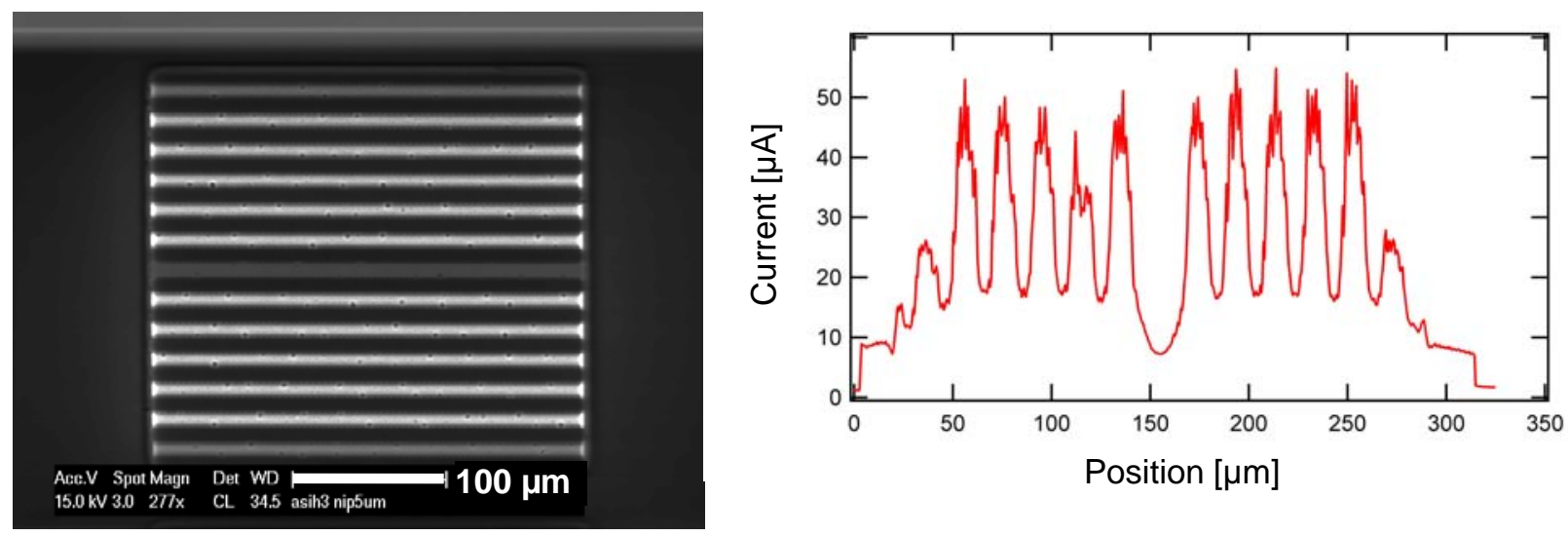

Figure 7. (Left) EBIC image of a set of microstrips with a width of $6.6 \mu \mathrm{m}$ and spacing of 13.4 $\mu \mathrm{m}$ covered with $5 \mu \mathrm{m}$ a-Si:H n-i-p diode at applied voltage of $30 \mathrm{~V}$ and beam energy of $20 \mathrm{keV}$ and (right) corresponding signal from EBIC line scans through the microstrip set. All strips are connected in parallel except the middle one.
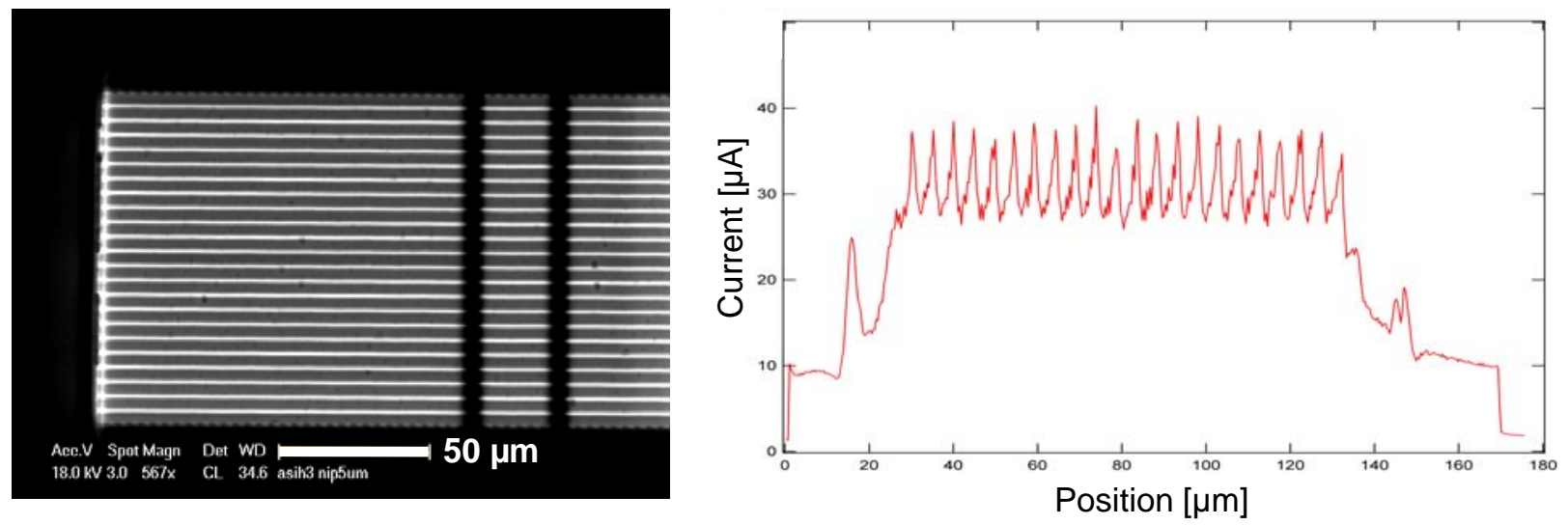

Figure 8. (Left) EBIC image of a set of microstrips with a width of $1.5 \mu \mathrm{m}$ and spacing of 3.5 $\mu \mathrm{m}$ covered with $5 \mu \mathrm{m}$ a-Si:H $5 \mu \mathrm{m}$ n-i-p diode at applied voltage of $30 \mathrm{~V}$ and beam energy of $20 \mathrm{keV}$ and (right) corresponding signal from EBIC line scans through the microstrip set. All strips are connected in parallel. Dark regions in the EBIC image of the strips correspond to degraded area by previous EBIC line scans. 
Effect of the a-Si:H diode thickness as well and configuration (n-i-p or M-i-p) are summarized in Table 2 for 2 different microstrip geometries (wide and narrow). One can see that thicker diodes (in the present case) increase the $S / N$ ratio while keeping the $L C C$ almost constant. Replacing the n-i-p configuration with $M-i-p$ helps to improve the $S / N$ ratio for large microstrips, but is not effective for narrow ones. Even though the average electric field was kept constant for all measurements, it is expected that the field distribution in the intrinsic layer may be quite different for the three types of diodes as discussed in the diode optimization section above. The applied voltage of $60 \mathrm{~V}$ for $10 \mu \mathrm{m}$ thick devices was probably not sufficient to achieve full depletion and could therefore result in a lower $I_{\text {strip }}$ and corresponding lower $S / N$.

Table 1. $S / N$ and $L C C$ values determined for various microstrip geometries (width and spacing) and a $5 \mu \mathrm{m}$ a-Si:H n-i-p diode.

\begin{tabular}{|c|c||c|c|c|}
\hline $\begin{array}{c}\text { Strip width } \\
{[\mu \mathrm{m}]}\end{array}$ & $\begin{array}{c}\text { Spacing } \\
{[\mu \mathrm{m}]}\end{array}$ & $\begin{array}{c}\text { Pitch } \\
{[\mu \mathrm{m}]}\end{array}$ & $S / N$ & LCC [\%] \\
\hline 0.6 & 1.4 & 2 & - & - \\
\hline 1.5 & 3.5 & 5 & $28.3 \pm 1.4$ & $62.0 \pm 0.3$ \\
\hline 3 & 7 & 10 & $18 \pm 1$ & $57.0 \pm 0.2$ \\
\hline \hline 6.6 & 13.4 & 20 & $39.0 \pm 1.7$ & $34.0 \pm 0.2$ \\
\hline 16.5 & 33.5 & 50 & $200 \pm 30$ & $11.5 \pm 0.1$ \\
\hline
\end{tabular}

Table 2. $S / N$ and LCC values determined for two microstrip geometries (width and spacing) and 3 different a-Si:H n-i-p diodes (with different thickness values and diode configuration).

\begin{tabular}{|c|c|l|c||c|}
\hline $\begin{array}{c}\text { Strip width } \\
{[\mu \mathrm{m}]}\end{array}$ & $\begin{array}{c}\text { Spacing } \\
{[\mu \mathrm{m}]}\end{array}$ & Diode structure & $\mathrm{S} / \mathrm{N}$ & LCC [\%] \\
\hline \multirow{3}{*}{16.5} & \multirow{3}{*}{33.5} & n-i-p $5 \mu \mathrm{m}$ & $200 \pm 30$ & $11.5 \pm 0.1$ \\
\cline { 3 - 5 } & & n-i-p $10 \mu \mathrm{m}$ & $1.2 \pm 0.3$ & $14.2 \pm 0.8$ \\
\cline { 3 - 5 } & \multirow{2}{*}{1.5} & M-i-p $10 \mu \mathrm{m}$ & $40 \pm 1$ & $7.4 \pm 0.4$ \\
\hline \hline \multirow{2}{*}{3.5} & n-i-p $5 \mu \mathrm{m}$ & $28.3 \pm 1.4$ & $62.0 \pm 0.3$ \\
\cline { 3 - 5 } & & n-i-p $10 \mu \mathrm{m}$ & $3.2 \pm 0.4$ & $80 \pm 1$ \\
\cline { 3 - 5 } & & M-i-p $10 \mu \mathrm{m}$ & $2.2 \pm 0.5$ & $80.6 \pm 1.5$ \\
\hline
\end{tabular}

\section{Microchanel plate detectors - fabrication}

For AMCP testing purposes, $15 \times 15 \mathrm{~mm}^{2}$ reticles have been defined with 24 pixels of $0.5 \times 0.5,1 \times 1$ and $2 \times 2 \mathrm{~mm}^{2}$ (see Fig. 9). Various reticles were designed with micro-channels (over each pixel) with a (nominal) diameter of 1.5 to $5 \mu \mathrm{m}$ separated by a gap of (nominally) 2.5 to 3.5 $\mu \mathrm{m}$. In Fig. 10, one can see SEM pictures of the AMCP surface of the corner of one pixel, as well as the cross section of some channel drilled by DRIE. Parallel channel walls can be obtained with high aspect ratios. 


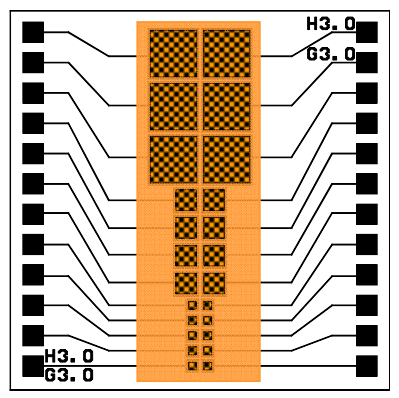

Figure 9. Schematic view of an AMCP test reticle. The back electrodes and bonding pads are in black; the colored/shaded area is covered by the thick a-Si:H layer while the patterned squares correspond to the area where the micro-channels are drilled.
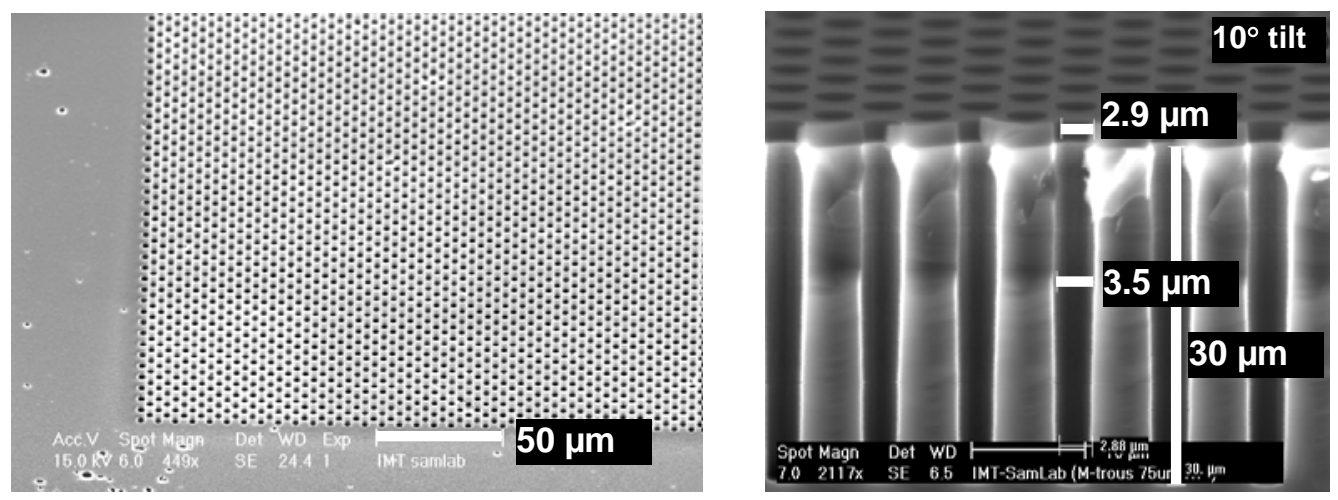

Figure 10. SEM pictures of the surface and cross section of thick a-Si:H layers with holes drilled by DRIE.

Fabrication of AMPC still results in the formation of local defects for some devices (see Fig. 11 and 12). In other devices an erosion of the front surface is sometime observed (Fig. 11 right). Even though both types of defects seem to take place at the end of the DRIE process, there is no direct link between the two as demonstrated by Fig. 12 where the surface is smooth and intact but local defects are present. Additional $\mathrm{SiO}_{\mathrm{x}}$ layer between the $\mathrm{Al}$ back contact and the intrinsic layer was introduced to improve the fabrication process.
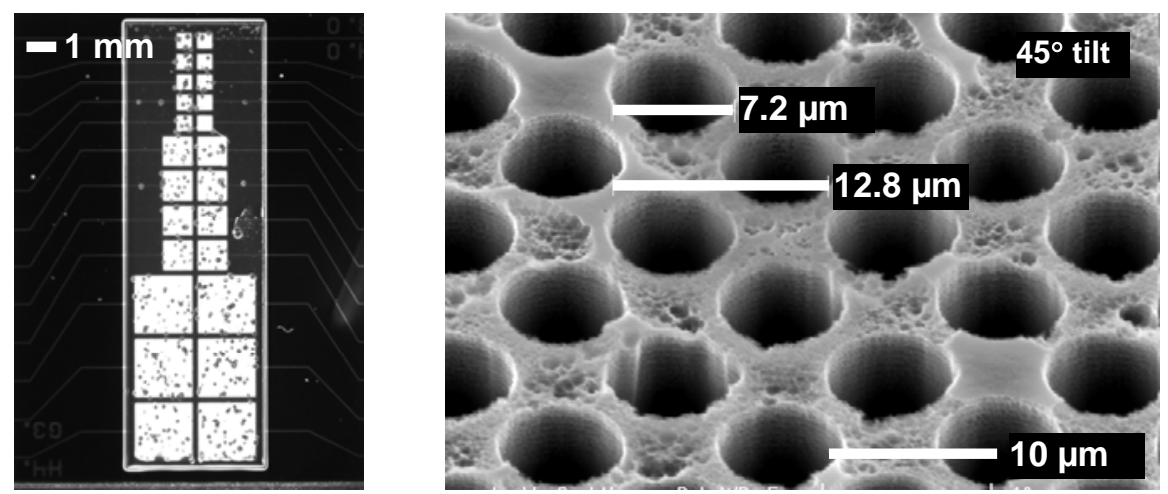

Figure 11. (Left) Picture of an AMCP reticle with many local defects on the pixel areas. (Right) SEM detail view of the pixel area showing the eroded surface. 


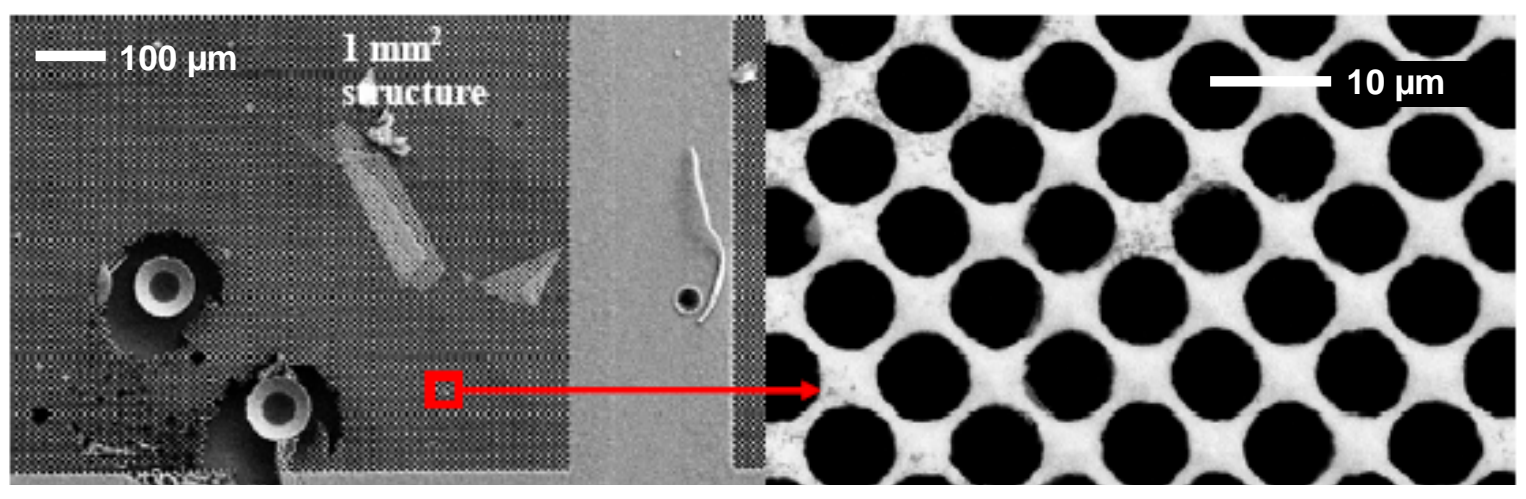

Figure 12. (Left) SEM picture of a pixel with two local defects and (Right) zoom over a defect free non-eroded part of the pixel.

\section{Microchanel plate detectors - characterization}

Preliminary measurements of AMCP already demonstrated multiplication of the primary electrons [11,12]. However, all AMCP reticles characterized in the past suffered from the erosion of the surface. resulting in etching of the top contact (a-Si:H n-layer), leading to very non uniform response of the microchannels over the pixel areas [11,12]. For devices without such erosion we can see that the response of each channel (see EBIC image in Fig. 13) is uniform. The local defects are clearly visible but do not contribute to the pixel response. If we zoom in (see Fig. 14 left) we can observe that only the periphery of the channel openings leads to EBIC signal, i.e. to a signal amplification given by the multiplication of the secondary electrons emitted from the impact of the EBIC electron beam with the channels. This result can be easily understood by looking at 4 possible interactions of the incoming electron beam with the microchannels (Fig. 15). Highest signal with uniform response of the entire channel opening is expected when the channel axis is tilted with respect to the incoming beam and this is what is observed experimentally (see Fig. 14 right).

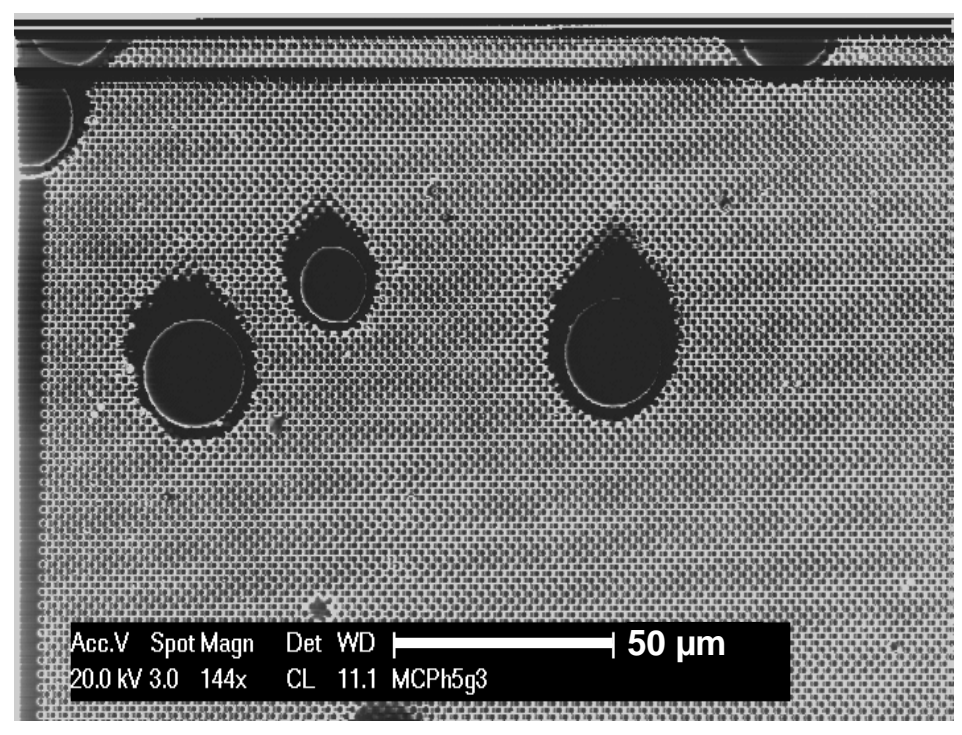

Figure 13. Partial EBIC image of a $1 \mathrm{~mm}^{2}$ pixel of AMCP with non-eroded top surface. 

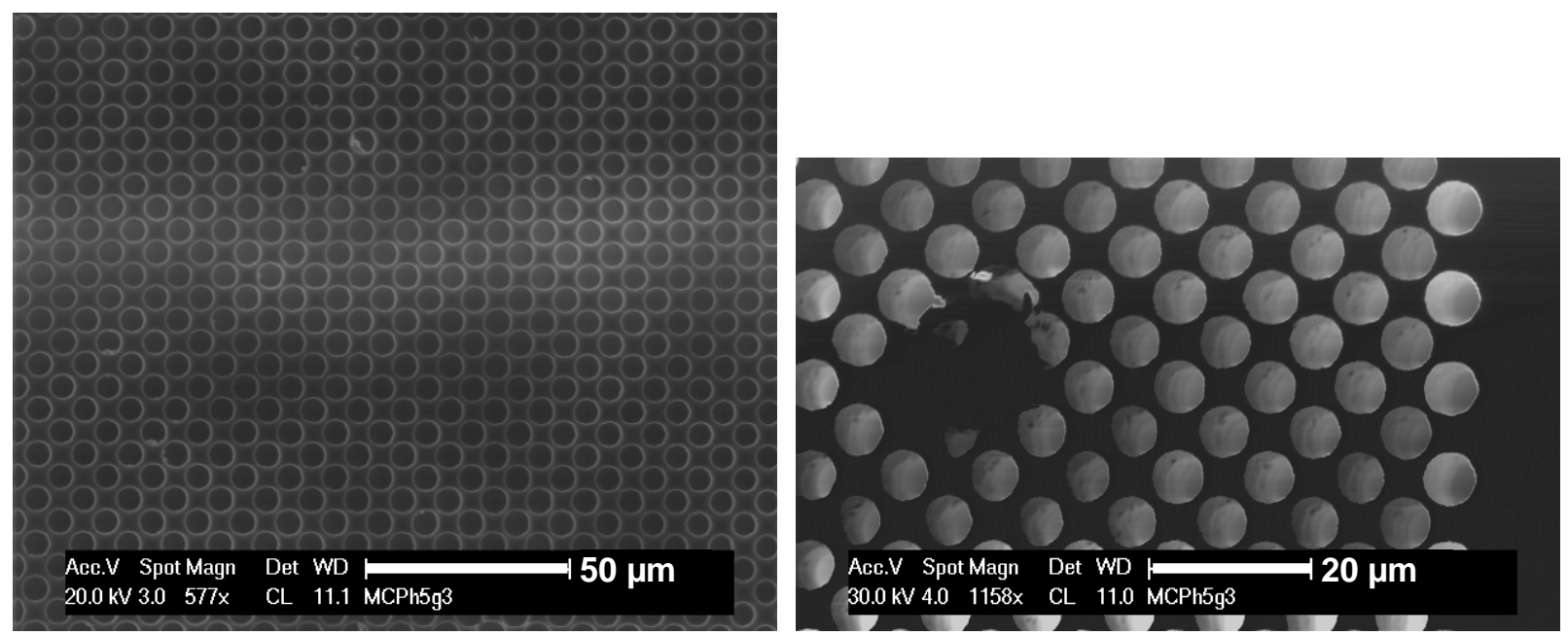

Figure 14. Detailed EBIC image of the microchannel openings for AMCP perpendicular to the electron beam (no tilt, left) and with $10^{\circ}$ tilt (right). Pictures are taken on the same pixel as in Fig. 13.

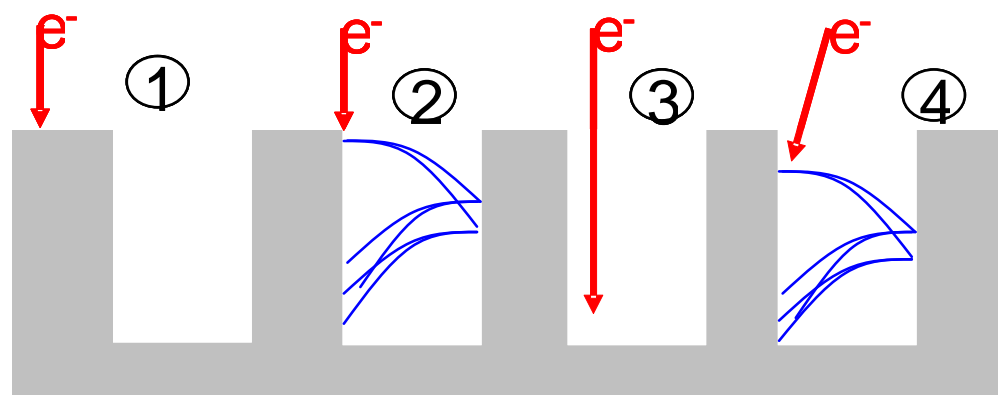

Figure 15. Possible interaction of an incoming electron with one microchannel. Only cases 2 and 4 lead to multiplication of the primary electrons. Such multiplication can only take place when the incoming electron hit the periphery of the channel opening or enter that opening with a certain angle.

\section{CONCLUSIONS}

Despite the fact that a-Si:H has not yet been implemented in any application for the direct detection of particles, we believe that this material remains very attractive when a large radiation hardness is required. Pixel or microstrip detectors involving a-Si:H thick diodes can provide unmatched spatial resolution and are suitable for very accurate beam monitoring or positioning. Such vertical integration would also be appropriate for high resolution particle imaging. However, detection of single minimum ionizing particle has not yet been achieved and may limit the potential of a-Si:H in high energy physics experiments. Further device improvement is here necessary.

a-Si:H is also a promising material candidate for MCP. In contrast to current MCP technology (using lead glass or c-Si wafer), a-Si:H has a bulk resistivity that enables fast channel charge replenishment. It allows less cumbersome fabrication process (compared to conventional 
MCP) and permits a vertical integration of MCP on the readout electronics. First AMCP were successfully fabricated and multiplication of secondary electrons has been observed as expected. Some processing issues have to be solved in order to optimize the device performance and get better understanding of AMCP potential and limitations.

\section{ACKNOWLEDGMENTS}

This work was partially supported by the Swiss National Science Foundation under Contract 200021_126926/1

\section{REFERENCES}

1. H. Schade, in “Thin Film Silicon Solar” edited by A. Shah. EPFL Press, 2010

2. V. Perez-Mendez et al., J. of Non-Cryst. Sol. 137\&138 (1991) 1291.

3. N. Kishimoto et al., J. Nucl. Mater. 258-263 (1998) 1908.

4. J. R. Srour et al., IEEE Trans. Nucl. Sci. 45 (1998) 2624.

5. V. P. Mendez et al., Nucl. Instrum. Methods Phys. Res. A273 (1988) 127.

6. Kim et al., Int. J. of Prec. Engin. and Manuf. 9 (2008) 86.

7. N. Wyrsch et al., MRS Proc. Symp. Vol. 869, 2005, 3-14.

8. M. Despeisse et al., IEEE Trans. Nucl. Sci. 55 (2008) 805.

9. C. Miazza et al., MRS Proc. Symp. Vol. 910 (2006) A17-03.

10. N. Wyrsch et al., Sensors 8 (2008) 4656.

11. F. Powolny, Ph.D. thesis, University of Neuchâtel, 2009.

12. N. Wyrsch et al., MRS Proc. Symp. Vol. 1245, 2010, 193.

13. J. L. Wiza, Nucl. Instr. and Meth. 162, 1979, 587-601.

14. C. P. Beetz et al., Nucl. Instr. and Meth. In Phys. Res. A 442, 2000, 443-451.

15. Q. Duanmu et al., Proc. of SPIE Vol. 4601, 2001, 284-287.

16 N. Wyrsch et al., MRS Proc. Symp. Vol. 869, 2005, 3-14.

17 http://www.gel.usherbrooke.ca/casino/What.html

18. N. Wyrsch et al., MRS Proc. Symp. Vol. 808 (2004) 441.

19. P.Cuony et al., Appl. Phys. Lett. 97 (2010) 213502.

20. P.Cuony et al., this volume. 